\title{
Essais
}

ESSAIS

Revue interdisciplinaire d'Humanités

$4 \mid 2014$

Éducation et humanisme

\section{Culture humaniste et enseignement en Italie : un héritage problématique}

\section{Maria Cristina Panzera}

\section{(2) OpenEdition}

1 Journals

Édition électronique

URL : https://journals.openedition.org/essais/10007

DOI : 10.4000/essais.10007

ISSN : 2276-0970

Éditeur

École doctorale Montaigne Humanités

\section{Édition imprimée}

Date de publication : 15 avril 2014

Pagination : 133-145

ISBN : 978-2-9544269-2-1

ISSN : 2417-4211

\section{Référence électronique}

Maria Cristina Panzera, "Culture humaniste et enseignement en Italie : un héritage problématique », Essais [En ligne], 4 | 2014, mis en ligne le 17 janvier 2022, consulté le 20 janvier 2022. URL : http:// journals.openedition.org/essais/10007; DOI : https://doi.org/10.4000/essais.10007 


\section{Culture humaniste et enseignement en Italie : un héritage problématique}

Maria Cristina Panzera

La dernière réforme en date de l'enseignement en Italie a été réalisée par Mariastella Gelmini, ministre du gouvernement Berlusconi de 2008 à 2011. Touchant tous les niveaux de l'éducation nationale, de l'école primaire, avec notamment le retour au maitre unique, aux universités, elle a été accompagnée et suivie par un cortège de manifestations, de polémiques ainsi que par de vastes débats médiatiques au sujet, entre autres, de l'adéquation entre l'offre de formation et la future insertion professionnelle des jeunes Italiens dans un contexte économique de crise. La contrainte économique a joué d'ailleurs un rôle indéniable dans la mise en ouvre de cette réforme, dont l'un des buts affichés était de procéder à ce qui dans le langage de la droite apparaissait comme une nécessaire "rationalisation » des dépenses en matière d'éducation, tandis que la gauche criait au sabotage de l'école publique. Si les mots sont parfois trompeurs, on pourra se référer aux chiffres publiés par le Ministère : plus de 8 milliards d'euros d'économie pour le budget de l'État, 150000 emplois supprimés (comprenant enseignants et personnel auxiliaire) ${ }^{1}$.

Selon les discours officiels, l'une des clés de la réforme a été l'exigence de moderniser le pays et de l'ouvrir aux progrès technologiques : " une nouvelle école qui marche avec son temps, où la qualité de l'enseignement prime sur la quantité des charges horaires ou sur la quantité excessive de matières $»^{2}$. De quoi relancer une vieille question nationale concernant le déséquilibre qui a toujours existé, en Italie, entre d'une part une culture humaniste largement favorisée pour la formation des élites et de l'autre une formation scientifique et technique jugée insuffisante. Quel avenir, au delà des polémiques, pour l'école italienne?

1 Le Trésor parle dans le Document d'économie et finance de 2011 d'une économie de 8 milliards et 13 millions d'euros, voir l'article de Claudio Tucci, "Istruzione: in tre anni tagli per 8 miliardi ", Il Sole 24 ore, 17 avril 2011.

2 "Una scuola nuova al passo con i tempi, in cui si privilegia la qualità dell'insegnamento rispetto alla quantità del carico orario e all'eccessivo numero di materie, come raccomandato dall'OCSE » (http://archivio.pubblica. istruzione.it/riforma_superiori). 
Pour examiner les dynamiques en cours, on partira dans un premier temps d'une présentation des nouveaux lycées issus de la réforme Gelmini, pour ce qui est notamment des programmes et des horaires de cours comparés avec le système français. Ensuite, une approche historique servira à une mise en perspective, afin de montrer en quoi la culture humaniste représente un héritage devenu problématique aujourd'hui. Revenant enfin à l'actualité, on verra que les initiatives de réforme doivent être mises en relation avec la logique qui préside désormais aux programmes d'évaluation internationale des systèmes éducatifs. Un équilibre difficile se dessine, alors, entre deux forces antinomiques, à savoir identité et traditions nationales d'un côté et, de l'autre, les tendances actuelles à l'européanisation, voire à la globalisation de l'enseignement supérieur.

\section{Les nouveaux lycées entre tradition et ouverture à la modernité}

La réorganisation des lycées est le pivot de la nouvelle réforme de l'enseignement en Italie. À côté des trois lycées traditionnels, qui sont par ordre d'ancienneté le liceo classico (filière littéraire), le lycée scientifique et le lycée linguistique, on assiste à la création de trois nouveaux lycées : lycée artistique, lycée musical, lycée des sciences humaines ${ }^{3}$. On touche une population scolaire conséquente, car on estime que les lycéens représentent $43 \%$ des élèves de l'enseignement secondaire, sachant qu'en 2007 l'âge de l'obligation scolaire a été porté à 16 ans. D'après les sites ministériels, la répartition des élèves de l'enseignement secondaire en fonction de leur origine scolaire est la suivante :

- lycées ( $43 \%$ des étudiants),

- instituts techniques (34\%),

- instituts professionnels (23\%).

Dans chacun de ces six lycées, tout est fixé en termes de volume horaire, choix de matières, programmes à suivre (dans le jargon ministériel : objectifs spécifiques d'apprentissage) et on met fin à toute une prolifération de parcours expérimentaux et d'options qui s'étaient développés à partir des années quatre-vingt, quand on avait cherché à adapter les trois lycées traditionnels à de nouvelles exigences pédagogiques. On peut reconnaître un principe anti-élitiste derrière l'extension du label "lycée " à des formations autres que les filières littéraire ou scientifique traditionnelles : notamment le lycée des sciences humaines et

3 Voici un choix de sites utiles sur l'école italienne que nous avons consultés pour ce chapitre : http://www.edscuola.it/archivio/norme/programmi/licei_2010.pdf (Programmes ministériels italiens)

http://www.education.gouv.fr/nouveau-lycee/index.php www.eurydice.org/ (Network on education systems and policies in Europe) http://www.invalsi.it/invalsi/ri/Pisa2009/documenti/OCSE-PISA2009_slide.pdf http://www.istruzione.it/web/hub/riforma_istruzione (Sur la réforme Gelmini) http://nuovilicei.indire.it/ (Les programmes ou "Objectifs spécifiques d'apprentissage »). 
sociales apparait comme une forme ennoblie de ce que représentait autrefois l'institut de ragioneria, l'école des comptables d'où sortaient les cadres moyens de l'administration et des banques.

La structure générale des lycées reste inchangée, elle couvre cinq ans à la fin de la scuola media, l'équivalent du collège français, pour aboutir à l'examen de maturità (tableau I).

Tableau I : système scolaire français et italien

\begin{tabular}{|l|l|l|}
\hline Âge & France & Italie \\
\hline 18 & & $5^{\circ}$ Maturità \\
\hline 17 & Terminale Bac & $4^{\circ}$ \\
\hline 16 & Première & $3^{\circ}$ \\
\hline 15 & Seconde & $2^{\circ}$ \\
\hline 14 & Troisième & $1^{\circ}$ Liceo \\
\hline 13 & Quatrième & Terza Media \\
\hline 12 & Cinquième & Seconda Media \\
\hline 11 & Sixième & Prima Media \\
\hline
\end{tabular}

Si l'on considère les matières et leurs volumes horaires, il apparaît que le lycée italien se caractérise par sa vocation généraliste, ce qui est particulièrement frappant si l'on compare le lycée scientifique italien à la filière $S$ du lycée français.

Tableau II : programmes du lycée scientifique en Italie

\begin{tabular}{|l|c|c|c|c|c|}
\hline Matières & I & II & III & IV & V \\
\hline Littér. italienne & 4 & 4 & 4 & 3 & 4 \\
\hline Latin & 4 & 5 & 4 & 4 & 3 \\
\hline Langue étrangère & 3 & 4 & 3 & 3 & 4 \\
\hline Histoire & 3 & 2 & 2 & 2 & 3 \\
\hline Géographie & 2 & & & & \\
\hline Philosophie & & & 2 & 3 & 3 \\
\hline Sciences & & 2 & 3 & 3 & 2 \\
\hline Physique & & & 2 & 3 & 3 \\
\hline Mathématiques & 5 & 4 & 3 & 3 & 3 \\
\hline Dessin & 1 & 3 & 2 & 2 & 2 \\
\hline EPS & 2 & 2 & 2 & 2 & 2 \\
\hline Religion & 1 & 1 & 1 & 1 & 1 \\
\hline Total & $\mathbf{2 5}$ & $\mathbf{2 7}$ & $\mathbf{2 8}$ & $\mathbf{2 9}$ & $\mathbf{3 0}$ \\
\hline
\end{tabular}

Comme il apparaît dans le tableau II, la durée d'études au lycée scientifique en Italie est de cinq ans. Pour effectuer la comparaison entre le système italien et son équivalent français, nous avons sélectionné dans le tableau III seulement les trois dernières années du lycée italien, pour les confronter, de manière certes un peu artificielle, avec les classes de Seconde, Première et Terminale $S$ en France. 
Tableau III : comparaison entre les deux lycées, italien et français

\begin{tabular}{|c|c|c|}
\hline Matières & Liceo Scientifico & Lycée S \\
\hline Latin & $11 \mathrm{~h}: 3$ ans $=\mathbf{3 , 6}$ & Option (max. 6h) \\
\hline Littér. nationale & (11) 3,6 & (8h) 2,6 \\
\hline Langues étrangères & (10) 3,3 & (14) 4,6 \\
\hline Philosophie & (8) 2,6 & (3+ options) 1 \\
\hline Histoire et Géographie & (7) 2,3 & (9) 3 \\
\hline Histoire de l'Art & T. 16,4 & facultatif \\
\hline Mathématiques & (9) 3 & $(14$ et +$) \mathbf{4 , 6}$ \\
\hline Physique & (8) 2,6 & $(11$ et +$) \mathbf{3 , 6}$ \\
\hline Autres sciences (SVT, dessin etc.) & (11) 3,6 & (13) 4,3 \\
\hline Religion/Éducation civique & (3) 3 & $(1 \mathrm{~h} 30) \mathbf{0 , 5}$ \\
\hline EPS & (6) 2 & $(6+$ options $) 2$ \\
\hline
\end{tabular}

Dans le tableau III, nous avons simplement additionné les volumes horaires hebdomadaires de chaque matière pour chaque année de cours (total donné entre parenthèses). Le total divisé par trois donne un volume horaire moyen sur les trois ans de cours considérés. Par exemple pour les langues étrangères un élève italien est sollicité au rythme de 3,3 heures par semaine au cours de ses trois dernières années de lycée ( $10 \mathrm{~h}$ de cours est la somme des horaires hebdomadaires des trois années). L’opération est compliquée par le fait que le système français prévoit beaucoup plus d'enseignements optionnels que son homologue italien. Nous avons donc tranché en choisissant d'examiner le cas d'un lycéen à profil fortement scientifique qui ne prendrait pas le latin en exploration ni comme matière facultative et qui choisirait les sciences en option ou comme matière de spécialité en terminale.

En définitive pour l'aire humaniste, le lycéen italien totalise 16,4 heures de cours par semaine (moyenne sur les trois ans), tandis que le lycéen français en aura 11,2 (mais un peu plus s'il choisit l'enseignement facultatif de latin). Il est donc clair que la culture humaniste pèse lourd sur l'emploi du temps des jeunes Italiens. La littérature nationale est une matière fondamentale tout au long du lycée italien, alors qu'en France elle n'est plus étudiée en classe de Terminale, après l'épreuve anticipée du baccalauréat à la fin de la Première. Une autre grande différence concerne l'enseignement du latin (langue et littérature) qui constitue en Italie un enseignement obligatoire. Après la réforme, il reste obligatoire dans le cursus du lycée classique et linguistique, mais les élèves du lycée scientifique pourront à présent choisir entre un parcours traditionnel avec le latin et une option "sciences appliquées " sans latin, de même que les étudiants du lycée des sciences humaines pourront éviter le latin en choisissant l'option économique et sociale. Pas de latin, en revanche, dans le lycée artistique et dans le lycée musical.

Pour le domaine scientifique, la comparaison est plus difficile : certes, les lycéens italiens ont moins de mathématiques ( $9 \mathrm{~h}$ contre 14h et plus en France), moins de physique ( $8 \mathrm{~h}$ contre $11 \mathrm{~h}$ ), mais on leur propose aussi plusieurs heures de chimie, de biologie et de dessin technique, pour un total de $11 \mathrm{~h}$. Pour le 
lycée français, la chimie est déjà comprise dans le volume horaire de physique (Physique et Chimie), mais il faut rajouter des matières telles que « Sciences de la Vie et de la Terre » ou "Sciences de l'ingénieur ", plus éventuellement les options. Nous ne considérons pas, pour le cas français, les TPE ni les heures d'accompagnement individualisé ( $2 \mathrm{~h}$ tous les ans, les enseignements de spécialité sont donc sûrement sous-évalués dans nos calculs). En définitive, le total pour l'aire scientifique serait donc de 9,2 pour l'Italie, de 12,5 pour la France (moyenne hebdomadaire sur les trois ans).

Petite parenthèse, enfin, pour l'enseignement de la religion, qui est dispensé au rythme d'une heure par semaine dans le lycée italien, alors qu'en France les élèves ont une demi-heure par semaine d'éducation civique. Bien sûr, il faut entendre par religion un enseignement qui ne correspond plus au catéchisme traditionnel.

Quant à la présence des matières scientifiques dans le liceo classico, la réforme a introduit une légère réduction horaire. Nous considérons encore une fois les trois dernières années de lycée, en donnant la somme du volume horaire hebdomadaire pour chaque matière scientifique. ${ }^{4}$

Tableau IV : les sciences au liceo classico

\begin{tabular}{|l|c|c|}
\hline Matières & Avant la réforme & Réforme Gelmini \\
\hline Mathématiques & 7 & 6 \\
\hline Physique & 5 & 7 \\
\hline Sciences & 9 & 6 \\
\hline Total & $\mathbf{2 1}$ & $\mathbf{1 9}$ \\
\hline
\end{tabular}

Les nouveaux programmes des lycées français prévoient pour la filière $\mathrm{L}$ beaucoup plus de souplesse dans l'élaboration des parcours : si l'on considère le cas d'un élève très intéressé par les matières scientifiques qui choisirait donc les mathématiques et les sciences comme matières d'exploration en seconde ou comme enseignements spécifiques en première, on arrive à un total de $20 \mathrm{~h}$ par semaine sur les trois ans ${ }^{5}$. Une vingtaine d'heures au total, pour récapituler, contre 28 au lycée scientifique italien, 38 dans la filière $S$ en France. Mais le volume horaire n'est qu'un faible indicateur, puisque les différences concernent également les méthodes, les approches, les évaluations : dans la filière littéraire, en Italie, l'enseignement scientifique reste une matière orale, qui généralement ne donne pas lieu à des contrôles écrits, demeurant plus axée sur la réflexion théorique que sur l'application et les pratiques.

L'analyse des programmes scolaires des lycées nouvellement réformés montre

4 Voir le cas spécifique du lycée Clemente Rebora de Rho (en Lombardie) :

http://www.liceorebora.it/

5 Pour le détail : en classe de seconde on propose $4 \mathrm{~h}$ de mathématiques, $3 \mathrm{~h}$ de physique, $1 \mathrm{~h} 30$ de SVT, 3h d'exploration en sciences. En classe de première L : 1 h30 de sciences, $3 \mathrm{~h}$ de mathématiques comme enseignement spécifique. En Terminale $4 \mathrm{~h}$ de mathématiques. 
le poids que la culture humaniste (langues anciennes, littérature, philosophie) continue d'exercer encore aujourd'hui, y compris dans les filières scientifiques, faisant de l'école supérieure italienne une exception dans le cadre européen. Dans les documents officiels, cette prééminence accordée à la culture humaniste est justifiée en termes pédagogiques : c'est une école qui voudrait apprendre à penser, plus encore qu’à savoir faire. Dans cet esprit, par exemple, un récent décret du Ministère affirme au sujet du lycée scientifique :

Le parcours du lycée scientifique vise l'étude du lien entre culture scientifique et tradition humaniste. Il favorise l'acquisition des connaissances et des méthodes propres aux mathématiques, à la physique et aux sciences naturelles ${ }^{6}$.

Des propos qui se retrouvent développés dans les objectifs spécifiques d'apprentissage pour le lycée scientifique, qui indiquent comment la formation scientifique au lycée permet à l'élève d'atteindre les résultats suivants :

1. Avoir acquis une formation culturelle équilibrée dans les deux domaines linguistique-historique-philosophique et scientifique ; comprendre les étapes fondamentales dans le développement de la pensée, y compris dans une dimension historique et les liens entre d'une part les méthodes de connaissance propres aux mathématiques et aux sciences expérimentales et de l'autre celles qui caractérisent la recherche de type humaniste. 2. Savoir saisir les rapports entre le raisonnement scientifique et la réflexion philosophique ${ }^{7}$.

\section{L'éducation supérieure italienne et son héritage historique}

Cette insistance sur le développement de la pensée comme étant un enjeu essentiel de l'éducation supérieure, tout comme le traditionalisme qui caractérise les programmes d'études en Italie représentent un héritage historique devenu aujourd'hui problématique, comme le prouve le débat sur l'enseignement du latin au lycée.

Selon les données publiées par MIUR $2006^{8}$ sur des statistiques se référant à l'année 2005 , le nombre d'élèves concernés par l'étude des langues classiques en Italie s'élève à 1006000 (sur un total de 2500 000), soit $41 \%$ des élèves de l'école supérieure'. Seulement trois autres pays en Europe ont gardé le latin comme enseignement obligatoire dans au moins une filière du second cycle :

6 Ministère de l'Instruction, de l'Université et de la Recherche, décret du 4 février 2010, article 8.

7 « 1 . Aver acquisito una formazione culturale equilibrata nei due versanti linguistico-storico-filosofico e scientifico; comprendere i nodi fondamentali dello sviluppo del pensiero, anche in dimensione storica, e i nessi tra i metodi di conoscenza propri della matematica e delle scienze sperimentali e quelli propri dell'indagine di tipo umanistico; 2 . saper cogliere i rapporti tra il pensiero scientifico e la riflessione filosofica ", http://nuovilicei.indire.it/.

8 Ministère de l'Instruction, de l'Université et de la Recherche.

9 Latino perché ? Latino per chi? Confronti internazionali per un dibattito, Gênes, Tipografia Araldica, 2008 (www.treelle.org), p. 35. 
l'Autriche, le Danemark et les Pays-Bas. Selon les données présentées par l'association Treelle, l'étude des langues classiques se répartit de la manière suivante en Europe : en Allemagne entre 5 et $8 \%$ des étudiants sont concernés ; en France le pourcentage pour le latin est de $19 \%$ d'élèves au collège et seulement $3 \%$ au lycée, pour le grec de $3 \%$ au collège, $1 \%$ pour le lycée. Un parallèle peut être établi enfin entre l'Italie et la Grèce qui est le seul pays en Europe où l'enseignement du grec ancien est obligatoire ${ }^{10}$.

Il est clair que des raisons symboliques président à cette permanence des langues anciennes dans certains pays comme l'Italie et la Grèce, en relation avec des problématiques identitaires et politiques. Renvoyant pour le cas français aux nombreuses études qui retracent l'histoire du déclin des humanités classiques dans le système scolaire national, nous rappellerons les étapes principales de l'avant-Gelmini pour le lycée italien ${ }^{11}$.

Au lendemain de la réunification du pays, avec la réforme Casati de 1859, le système éducatif en vigueur en Piémont (royaume de Sardaigne, sous la couronne de Savoie) est appliqué partout dans le nouveau Royaume d'Italie ${ }^{12}$. Les programmes sont repris de la ratio studiorum des jésuites, l'enjeu étant de soustraire à l'Église la formation des futurs citoyens selon un idéal d'indépendance hérité de l'époque napoléonienne. C'est d'ailleurs Napoléon qui par décret impérial a procédé le 18 octobre 1810 à la création de l'École Normale Supérieure de Pise dans ce même but de contrer la mainmise des écoles religieuses sur la formation des élites intellectuelles du pays.

En 1923, le philosopheet ministre del'Éducation nationale Giovanni Gentile, avec sa réforme des lycées (la plus fasciste de ses réformes, comme le disait Mussolini), place la culture humaniste au centre de la formation des élites ${ }^{13}$. Il prolonge la scolarité obligatoire jusqu’à l'âge de quatorze ans et fixe notamment

10 Ibid.

11 Françoise Waquet, Le latin ou l'empire d'un signe (XVI'-XX'e siècles), Paris, Albin Michel, 1998 et Jean Leduc, "La place du latin et du grec dans les cursus de l'enseignement secondaire en France de 1902 à nos jours", in Les Humanités pour quoi faire : enjeux et propositions, colloque international Université Toulouse II-Le Mirail, IUFM Midi-Pyrénées, 27-29 mai 2010. En 1962, avec la création de l'école unique, gratuite et obligatoire pour les 11-13 ans, le latin reste une matière d'étude en deuxième année, optionnelle en troisième année mais obligatoire pour l'accès au lycée. Le recul du latin commence dans les années 1960-1970, à partir du moment où sa connaissance n'est plus demandée pour l'accès à l'Université. La disparition du latin en sixième date du 9 octobre 1968, à l'initiative du ministre de l'Éducation nationale Edgar Faure. Avec le nouveau contrat pour l'école de François Bayrou en 1995 le latin est de nouveau introduit comme option en cinquième.

12 Angelo Semeraro, Il sistema scolastico italiano. Profilo storico, Rome, La Nuova Italia scientifica, 1997.

13 Emma Giammattei, "Critica e filosofia. Croce e Gentile ", in Storia della Letteratura italiana, vol. VIII. Tra l'Otto e il Novecento, Rome, Salerno, p. 967-1016, p. 998 sur Gentile et le marxisme. Voir aussi Badaloni, Nicola et Muscetta, Carlo, Labriola, Croce, Gentile, Bari, Laterza, 1981. 
les programmes d'étude des lycées qui sont restés pour l'essentiel inchangés jusqu'à présent. Gentile, ancien élève de l'École Normale Supérieure de Pise, était comme Benedetto Croce, philosophe idéaliste et, comme l'a bien souligné Sergio Romano : "La réforme conçue par Gentile était en parfaite harmonie avec sa conception de l'État éthique car elle attribuait à ce dernier la responsabilité morale de l'éducation et se proposait finalement la formation d'une société organique, dominée au sommet par une caste de citoyens philosophes $»^{14}$.

Dans la conception de Gentile, la voie royale vers l'université était représentée par le lycée classique, le seul qui permettait de s'inscrire à la Faculté de Droit ou à celle de Lettres et philosophie. L'accès aux études universitaires était assez limité pour les jeunes diplômés des instituts techniques, qui pouvaient choisir uniquement entre les facultés d'agronomie, d'économie et commerce ou de sciences statistiques. Un système hiérarchique bien établi, en définitive, qui était fondé sur un principe de sélection sociale reflétant également une hiérarchie entre les différentes disciplines : au sein de l'enseignement unique " Histoire et Philosophie ", cette dernière en venait notamment à occuper un rôle central parce qu'elle représentait l'aboutissement d'une formation amenant les individus à la prise de conscience de l'Esprit et à l'exercice de la liberté (Angelo Semeraro renvoie, à ce propos, à la réforme prussienne de 1810 menée par Wilhelm von Humboldt ${ }^{15}$ ). Une logique de progression établissait l'accès à la philosophie au lycée comme une seconde étape venant après l'étude de la religion que Gentile réservait à l'école primaire en guise de première ouverture aux idées abstraites et spirituelles. Les différents parcours étaient déjà différenciés à la sortie de l'école élémentaire puisque la scuola media unique, l'équivalent du collège français, est une création de 1939, sous le ministre Bottai ${ }^{16}$.

Dans ce cadre, la dévalorisation des disciplines scientifiques s'explique par opposition au matérialisme incarné par les deux courants que sont le positivisme, qui avait réussi à s'imposer dans les universités italiennes au début du siècle, et le marxisme dont Gentile, de même que Croce, avait étudié et renié les fondements. De ce point de vue, il a été dit que la formation technique dans l'enseignement supérieur dessiné par Gentile représentait même un retour en arrière par rapport aux innovations de la réforme de Casati. En effet, les écrits pédagogiques de Gentile offrent aux lecteurs plusieurs remarques assez méprisantes envers les savoirs scientifiques. Pour le philosophe idéaliste, les activités de l'esprit et la dynamique qui amène la pensée à se traduire en acte ne sauraient se résoudre aux simples tâches stériles de la raison scientifique : "cette science qui par le truchement de ses schémas, de ses formules, de ses tracés, de ses potions,

14 Sergio Romano, "Giovanni Gentile, philosophe du fascisme », in Vingtième siècle. Revue d'histoire, 21 (1989), p. 71-82, ici p. 74.

15 A. Semeraro, op. cit., p. 63.

16 Ibid., p. 70. 
de ses cadavres, de ses plantes desséchées et de ses bêtes empaillées est comme un monde de spectres où l'âme ressent le froid de la mort $"^{17}$.

Les idées d'un autre grand représentant de l'idéalisme néo-hégélien dominant à l'époque, Benedetto Croce, confirment le rôle subordonné qu'occupent les sciences au sein de cette pédagogie. Dans sa Philosophie comme science de l'esprit (quatre volumes publiés entre 1902 et 1917) Croce distingue à l'intérieur de l'Esprit les deux moments que sont l'exercice de la théorie et la sphère pratique : "Par la théorie l'homme comprend les choses et par la pratique il intervient sur celles-ci ${ }^{18}$. L'intuition et l'intelligence sont au service de l'activité théorique dans les domaines respectivement de l'esthétique et de la logique, tandis que les deux moments de l'Esprit pratique sont l'économie et la morale, présidant respectivement à la recherche de l'utile et du bon. Les sciences naturelles et les mathématiques ont recours à des pseudo-concepts abstraits dont l'utilité se limite à la sphère pratique et que Croce tient à distinguer des concepts purs figurant à la base de la logique, par exemple le concept de substance ou de changement (voir Logica come scienza del concetto puro, publié en 1909). Dans l'un de ses derniers essais au sujet de Hegel, en 1952, Croce écrivait : " Les sciences naturelles et les disciplines mathématiques ont cédé de bon gré à la philosophie le privilège de la vérité et sur le ton de la résignation, voire en souriant, elles avouent que leurs concepts sont des concepts de service et d'utilité pratique et qu'ils n’ont rien à voir avec la méditation du vrai $»^{19}$.

Pour résumer, le traditionalisme du système éducatif italien s'explique par une multiplicité de facteurs qui sont à la fois d'ordre idéologique et politique. L'influence profonde de l'idéalisme philosophique a cautionné le maintien des disciplines humanistes, philosophie, littérature, langues anciennes, au sommet des modèles culturels de l'élite. On pourra évoquer encore la force du mythe identitaire de la romanité que l'orgie de classicisme fasciste n'a sans doute pas réussi à effacer complètement ${ }^{20}$, sans oublier l'hostilité qu'a toujours manifestée l'Église, en Italie, envers les dangers matérialistes, y compris dans le domaine des sciences. La continuité des élites et des cadres de l'administra-

17 «[... quella scienza che coi suoi schemi, le sue formule, le sue leggi, i suoi tracciati, i suoi preparati, i suoi cadaveri e le sue piante disseccate e le sue bestie impagliate è come un mondo di spettri, dove l'anima sente il freddo della morte ", G. Gentile, Sommario di pedagogia generale, Bari, Laterza, vol. I, p. 230.

18 "Con la forma teoretica l'uomo comprende le cose, con la pratica le vien mutando ", B. Croce, Estetica come scienza dell'espressione, Milano, Palermo, Napoli, 1902, p. 14.

19 "Le scienze naturali e le discipline matematiche, di buona grazia, hanno ceduto alla filosofia il privilegio della verità, ed esse rassegnatamente, o addirittura sorridendo, confessano che i loro concetti sono concetti di comodo e di pratica utilità, che non hanno niente da vedere con la meditazione del vero ", B. Croce, Indagini su Hegel e schiarimenti filosofici, Bari, Laterza, 1967, p. 283.

20 Rosario Drago a attiré l'attention par exemple sur une certaine faiblesse de l'anti-fascisme italien qui a exercé sa critique plus sur la République de Salò que sur les formes de la vie intellectuelle et de l'éducation élaborées sous le régime, cf. Latino perché? Latino per chi?, op. cit., p. 100. 
tion publique dans l'après-guerre a été également pointée comme étant une cause structurelle de conservatisme culturel, tout comme l'impact modéré de mai 68 sur la société italienne. Une dernière confirmation vient des statistiques élaborées par Carboni en 2007 concernant les élites italiennes à partir des données publiées dans le volume Who is who in Italy, 2005. Il en résulte qu'un sixième est représenté par des diplômés de facultés humanistes, lesquels constituent moins d'un sixième du nombre total des laureati. Un quart des élites est composé par des cadres issus de facultés scientifiques et économiques, avec une baisse par rapport à $1990^{21}$.

\section{Les enjeux de la (post)modernité}

Peuple de poètes... Avec son lourd héritage et son fardeau de stéréotypes l'école italienne se retrouve à devoir se mesurer aujourd'hui avec la modernité et le nouveau cadre de compétitivité internationale associée au processus d'européanisation. Que l'on pense aux programmes PISA (Program for International Student Assessment), émanation de l'OCDE (Organisation pour la Coopération et le Développement Économique, OCSE en Italie) ${ }^{22}$. Ce programme produit à échéance triennale des statistiques sur les niveaux de connaissance des lycéens en Europe et dans le reste du monde. À lire les lourds dossiers de synthèse et les commentaires accompagnant ces campagnes de tests, on aurait envie de dire que l'histoire a bien su venger les disciplines scientifiques et techniques du mépris si longtemps enduré :

De plus en plus d'éléments montrent que par rapport à un individu "normal ", les individus hautement qualifiés génèrent des externalités relativement importantes en matière de création et d'exploitation de connaissances, ce qui suggère qu'investir dans l'excellence peut profiter à toute la société (Minne et al., 2007). Cela s'explique notamment par le fait que les individus hautement qualifiés innovent dans divers domaines (organisation, marketing, design etc.) et que le fruit de leur innovation est bénéfique pour toute la société ou stimule le progrès technologique ${ }^{23}$.

Comme ce passage l'exprime bien, il est clair - n'en déplaise aux individus " autrement » qualifiés - que la qualité de l'éducation supérieure aujourd'hui se mesure à l'aune de la productivité, de l'utile et de l'innovation technologique. Toujours est-il qu'en matière de compétences scientifiques et mathématiques chez les jeunes de 15 ans, qui constituent la cible des programmes PISA, la vieille Europe s'est découverte, à travers ces tests, moins compétitive que des pays asiatiques comme la Corée et le Japon ou des pays où l'histoire

21 Carlo Carboni, Élite e classi dirigenti in Italia, Bari, Laterza, 2007, p. 27. Ce phénomène social est analysé également par Ernesto Galli della Loggia, L’identità italiana, Bari, Laterza, 1998.

$22 \mathrm{Pisa}^{\mathrm{TM}}$, 2006. Les compétences en sciences, un atout pour réussir, en ligne : www.oecd.org. Un autre site utile : http://www.invalsi.it/invalsi/ri/Pisa2009/documenti/OCSE-PISA2009_slide.pdf.

23 Les compétences en sciences, un atout pour réussir, vol. 1, p. 56. 
culturelle est plus récente, comme l'Australie, le Canada, la Nouvelle Zélande. En Europe, la Finlande et les Pays-Bas font encore figure de bons élèves, la France obtient des résultats honorables, mais les scores des jeunes Italiens sont inférieurs à la moyenne. En regardant dans les détails ces résultats, on remarque encore une fois l'élitisme de l'enseignement supérieur italien. Les comptes-rendus de l'OCSE pour l'Italie ont la caractéristique de proposer une micro-analyse où les moyennes nationales sont décomposées en fonction des particularités régionales : on remarque que les lycées de l'Italie du Nord fournissent des élèves mieux préparés pour passer ces tests avec succès (tableau $\mathrm{V}$ ).

Tableau V : Notes moyennes en sciences en fonction des aires géographiques en Italie

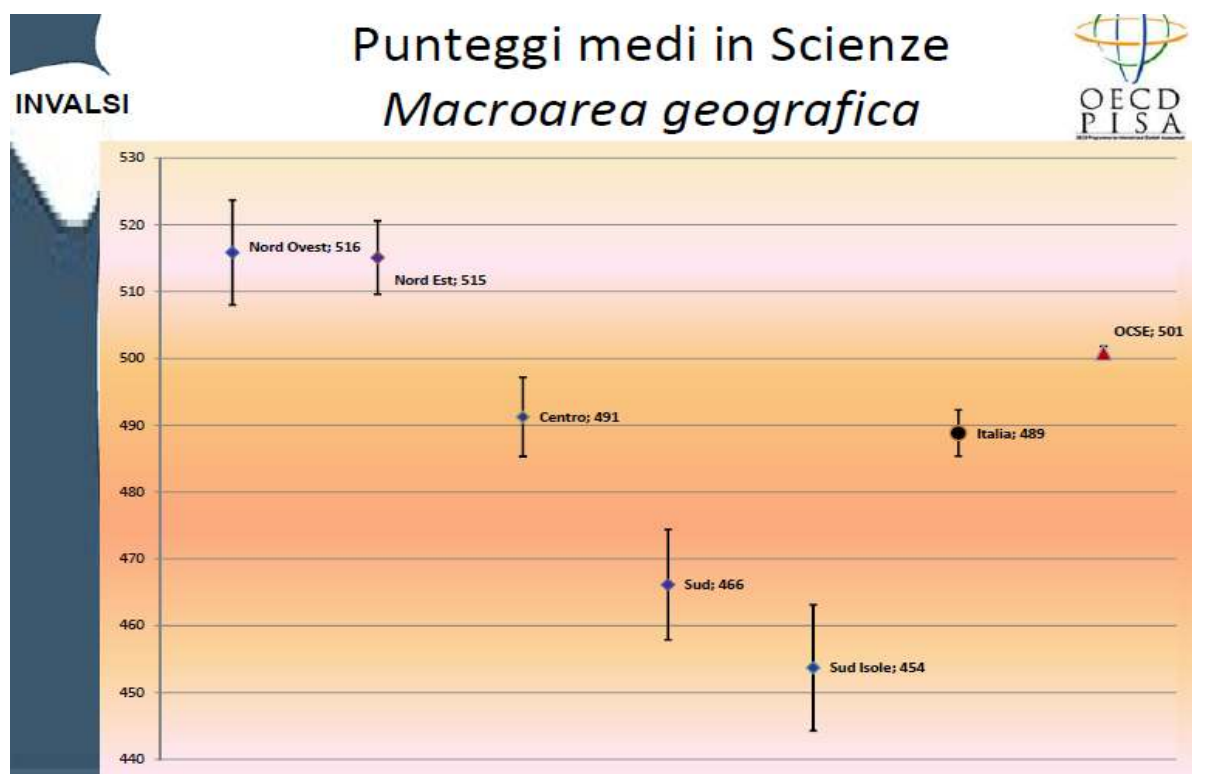

Autrement dit, la variable géographique entre Nord et Sud du pays est encore très sensible. Mais il faut compter également avec la variable sociologique : les performances des élèves du lycée sont nettement supérieures à celles des jeunes inscrits dans les instituts professionnels.

Cela signifie donc que les élites du pays rejoignent et même dépassent le niveau de formation des élites européennes, mais qu'on délaisse en bas de l'échelle 30 à $40 \%$ de la population juvénile. En effet, l'un des constats en matière de politique scolaire qui a émergé de l'ensemble de ces évaluations internationales est que l'équilibre et l'homogénéité dans la formation des jeunes, que ce soit au lycée ou dans les instituts professionnels, représente une clé de réussite : c'est le cas notamment pour la Finlande qui offre à ses jeunes l'égalité des chances. 
Au terme de cette analyse, il est évident que pour l'heure le système italien continue de représenter un cas unique en Europe. On comprend que la dernière réforme de la ministre Gelmini ait pu être qualifiée de néo-gentilienne par l'opposition, puisqu'elle était l'émanation d'un gouvernement de droite, qu'elle a été globalement plus attentive à l'organisation des lycées qu'aux problèmes liés à l'éducation technique et professionnelle, sans parvenir, enfin, à franchir le pas de la réduction des heures de latin dans les programmes des filières d'excellence. De fait, cette réforme a réalisé une sorte de compromis, tout en amorçant le processus qui va sans doute conduire à une progressive réduction de la culture humaniste dans les lycées, puisqu'on peut déjà prévoir dans les années à venir un abandon généralisé du parcours traditionnel avec latin au lycée scientifique, voire peutêtre l'exode du vieux liceo classico en direction des nouveaux lycées. En comparaison avec le lycée français et son fonctionnement à options, le système italien apparait certes plus rigide, puisque chaque parcours reste associé à un établissement particulier, avec son petit monde à part, les littéraires, les scientifiques, les artistes, les musiciens, etc., autant de cloisons qui alimentent une culture de la différence et du clan. Difficile, dans ce contexte, de dépasser vraiment les stéréotypes associés à l'élitisme. Quant à la question de la compétitivité, on ne saurait certes mettre naïvement sur le compte de l'éducation nationale, ni même de l'idéalisme philosophique qui l'a alimentée par le passé, la responsabilité de la crise profonde qui touche l'insertion professionnelle des jeunes Italiens depuis quelques décennies. D'autres responsabilités, bien plus graves, pèsent sur les récents protagonistes de la vie politique du pays, à tel point que la première question à poser en matière d'éducation concerne plutôt la formation de l'homo politicus et du citoyen. Ce serait une autre enquête à mener. Mais pour en rester à notre réflexion sur la réforme des lycées, on constate que malgré ses aspects plutôt modérés que nous avons essayé de détailler, des cris d'alarme se multiplient de la part de plusieurs intellectuels et universitaires italiens pour appeler à préserver le patrimoine culturel humaniste du pays $^{24}$. La question qui se pose plus que jamais, aujourd'hui, concerne, en effet, l'avenir des cultures nationales dans le processus d'européanisation en cours. En ce sens, le retard du système éducatif italien, ou plutôt son altérité, pourrait s'avérer paradoxalement utile, en termes de dialectique, pour la recherche commune de solutions équilibrées entre les différentes forces en jeu.

Maria Cristina Panzera EA 4593 CLARE

Université Bordeaux Montaigne Cristina Panzera@u-bordeaux-montaigne.fr

24 Giuseppe Cambiano, Perché leggere i classici, Bologne, Il Mulino, 2011. 


\section{Résumé}

Le traditionalisme du système éducatif italien s'explique par une multiplicité de facteurs d'ordre idéologique et politique. La réorganisation des lycées à l'intérieur de la récente réforme de l'enseignement réalisée par la ministre Gelmini (de 2008 à 2011) confirme la place importante accordée à la culture humaniste dans le pays. La comparaison avec le lycée français et la prise en compte des programmes d'évaluation internationale des élèves du secondaire (PISA) font ressortir la spécificité du système italien.

\section{Mots-clés}

Réforme des lycées, élites culturelles, Gentile, néo-hégélisme, évaluation internationale PISA.

\section{Abstract}

Traditionalism is a well known aspect of the Italian educational system. This has several ideological and political reasons. The reorganization of secondary school as a part of the reform accomplished by the minister M.S. Gelmini (between 2008 and 2011) confirms the important place which the humanistic culture has in this country. In this paper we compare the Italian and French high school systems. We then consider the results of the Program for International Student Assessment (PISA) in order to point out the specificities of the Italian system.

\section{Keywords}

High school reform, cultural elites, Gentile, Neo-Hegelianism, Program for International Student Assessment (PISA). 